\title{
REE From EOF
}

\author{
Eylee Jung ${ }^{1}$ and DaeKil Park ${ }^{1,2 *}$ \\ ${ }^{1}$ Department of Electronic Engineering, \\ Kyungnam University, Changwon 631-701, Korea \\ ${ }^{2}$ Department of Physics, Kyungnam University, Changwon 631-701, Korea
}

\begin{abstract}
It is well-known that entanglement of formation (EOF) and relative entropy of entanglement (REE) are exactly identical for all two-qubit pure states even though their definitions are completely different. We think this fact implies that there is a veiled connection between EOF and REE. In this context, we suggest a procedure, which enables us to compute REE from EOF without relying on the converse procedure. It is shown that the procedure yields correct REE for many symmetric mixed states such as Bell-diagonal, generalized Vedral-Plenino, and generalized Horodecki states. It also gives a correct REE for less symmetric Vedral-Plenio-type state. However, it is shown that the procedure does not provide correct REE for arbitrary mixed states.
\end{abstract}

\footnotetext{
*dkpark@kyungnam.ac.kr
} 


\section{INTRODUCTION}

Entanglement of formation (EOF) [1] and relative entropy of entanglement (REE) [2, 3] are two major entanglement monotones for bipartite systems. For pure states $\rho=|\psi\rangle\langle\psi|$ the $\operatorname{EOF} \mathcal{E}_{F}(\rho)$ is defined as a von Neumann entropy of its subsystem $\rho_{A}=\operatorname{tr}_{B} \rho$. On the contrary, REE is defined as minimum value of the relative entropy with separable states;

$$
\mathcal{E}_{R}(\rho)=\min _{\sigma \in \mathcal{D}} \operatorname{tr}(\rho \ln \rho-\rho \ln \sigma)
$$

where $\mathcal{D}$ is a set of separable states ${ }^{1}$. It was shown in Ref. [3] that $E_{R}(\rho)$ is a upper bound of the distillable entanglement[1]. The separable state $\sigma_{*}$, which yields a minimum value of the relative entropy is called the closest separable state (CSS) of $\rho$. Surprising fact, at least for us, is that although definitions of EOF and REE are completely different, they are exactly same for all pure states [3]. This fact may indicate that they are related to each other although the exact connection is not revealed yet. The main purpose of this paper is to explore the veiled connection between EOF and REE.

For mixed states $\rho$ EOF is defined via a convex-roof method[1, 5];

$$
\mathcal{E}_{F}(\rho)=\min \sum_{i} p_{i} \mathcal{E}_{F}\left(\rho_{i}\right)
$$

where the minimum is taken over all possible pure-state decompositions with $0 \leq p_{i} \leq 1$ and $\sum_{i} p_{i}=1$. The ensemble that gives the minimum value in Eq. 1.2 is called the optimal decomposition of the mixed state $\rho$. Thus, the main task for analytic calculation of EOF is derivation of an optimal decomposition of the given mixture. Few years ago, the procedure for construction of the optimal decomposition was derived [6, 7] in the two-qubit system, the simplest bipartite system, by making use of the time-reversal operation of spin-1/2 particles appropriately. In these references the relation

$$
\mathcal{E}_{F}(C)=h\left(\frac{1+\sqrt{1-C^{2}}}{2}\right)
$$

is used, where $h(x)$ is a binary entropy function $h(x)=-x \ln x-(1-x) \ln (1-x)$ and $C$ is called the concurrence. This procedure, usually called Wootters procedure, was re-examined

\footnotetext{
${ }^{1}$ Since REE is defined through another separable state $\sigma$, it is called "distance entanglement measure". Another example of the distance entanglement measure is a geometric entanglement measure defined as $E_{g}(\psi)=1-P_{\max }$, where $P_{\max }$ is a maximal overlap of a given state $|\psi\rangle$ with the nearest product state 4 .
} 
in Ref. [5] in terms of antilinearity. Introduction of antilinearity in quantum information theory makes it possible to derive concurrence-based entanglement monotones for tripartite [8] and multipartite systems [9]. Due to the discovery of the closed formula for EOF in the two-qubit system, EOF is recently applied not only to quantum information theory but also to many scientific fields such as life science[10].

While EOF is used in various areas of science, REE is not because of its calculational difficulty. In order to obtain REE analytically for given mixed state $\rho$ one should derive its CSS, but still we don't know how to derive CSS[11] even in the two-qubit system except very rare cases [3, 13, 14]. In Ref.[13] REE for Bell-diagonal, generalized Vedral-Plenio[3], and generalized Horodecki states [15] were derived analytically through pure geometric arguments 12 .

Due to the notorious difficulty some people try to solve the REE problem conversely. Let $\sigma_{*}$ be a two-qubit boundary states in the convex set of the separable states. In Ref.[16] authors derived entangled states, whose CSS are $\sigma_{*}$. This converse procedure is extended to the qudit system[17] and is generalized as convex optimization problems[18]. However, as emphasized in Ref.[13] still it is difficult to find a CSS $\sigma_{*}$ of given entangled state $\rho$ although the converse procedure may provide some useful information on the CSS[14].

In this paper we will try to find a CSS for given entangled two-qubit state without relying on the converse procedure. As commented, EOF and REE are identical for bipartite pure states although they are defined differently. This means that they are somehow related to each other. If this connection is unveiled, probably we can find CSS for arbitrary two-qubit mixed states because we already know how to compute EOF through Wootters procedure. To explore this issue is original motivation of this paper. We will show in the following that REE of many mixed symmetric states can be analytically obtained from EOF if one follows the following procedure:

(1) For entangled two-qubit state $\rho$ let $\rho=\sum_{j} p_{j} \rho_{j} \quad\left(\rho_{j}=\left|\psi_{j}\right\rangle\left\langle\psi_{j}\right|\right)$ be an optimal decomposition for calculation of EOF.

(2) Since $\rho_{j}$ are pure states, it is possible to obtain their CSS $\sigma_{j}$. Thus, it is straight to derive a separable mixture $\tilde{\sigma}=\sum_{j} p_{j} \sigma_{j}$.

(3) If $\tilde{\sigma}$ is a boundary state in the convex set of separable states, the procedure is terminated with $\sigma_{*}=\tilde{\sigma}$. 
(4) If $\tilde{\sigma}$ is not a boundary state, we consider $\pi=q \rho+(1-q) \tilde{\sigma}$. By requiring that $\pi$ is a boundary state, one can fix $q$, say $q=q_{0}$. Then we identify $\sigma_{*}=q_{0} \rho+\left(1-q_{0}\right) \tilde{\sigma}$.

This procedure is schematically represented in Fig. 1.

In order to examine the validity of the procedure we have to apply the procedure to the mixed states whose REE are already known. Thus, we will choose the Bell-diagonal, generalized Vedral-Plenio and generalized Horodecki states, whose REE were computed in Ref. [3, 13, 16] through different methods. Also, we will apply the procedure to the less symmetric mixed states such as Vedral-Plenio-type and Horodecki-type states whose REE were computed in Ref.[14] by making use of the the converse procedure introduced in Ref.[16].

The paper is organized as follows. In section II we show that the procedure generates the correct CSS for Bell-diagonal states. In section III and section IV we show that the procedure generates the correct CSS for generalized Vedral-Plenio and generalized Horodecki states, respectively. In section $\mathrm{V}$ we consider two less symmetric states, Vedral-Plenio-type and Horodecki-type states. It is shown that while the procedure generates a correct CSS for the former, it does not give a correct one for the latter. In section VI a brief conclusion is given. In appendix we prove that EOF and REE are identical for all pure states by making use of the Schmidt decomposition. The Schmidt bases derived in this appendix are used in the main body of this paper.

\section{BELL-DIAGONAL STATES}

In this section we will show that the procedure mentioned above solves the REE problem of the Bell-diagonal states:

$$
\rho_{B D}=\lambda_{1}\left|\beta_{1}\right\rangle\left\langle\beta_{1}\left|+\lambda_{2}\right| \beta_{2}\right\rangle\left\langle\beta_{2}\left|+\lambda_{3}\right| \beta_{3}\right\rangle\left\langle\beta_{3}\left|+\lambda_{4}\right| \beta_{4}\right\rangle\left\langle\beta_{4}\right|
$$

where $\sum_{j=1}^{4} \lambda_{j}=1$, and

$$
\begin{array}{rlrl}
\left|\beta_{1}\right\rangle & =\frac{1}{\sqrt{2}}(|00\rangle+|11\rangle) & \left|\beta_{2}\right\rangle=\frac{1}{\sqrt{2}}(|00\rangle-|11\rangle) \\
\left|\beta_{3}\right\rangle=\frac{1}{\sqrt{2}}(|01\rangle+|10\rangle) & \left|\beta_{4}\right\rangle=\frac{1}{\sqrt{2}}(|01\rangle-|10\rangle) .
\end{array}
$$

The CSS and REE of $\rho_{B D}$ were obtained in many literatures [3, 13, 14] through various different methods. If, for convenience, $\max \left(\lambda_{1}, \lambda_{2}, \lambda_{3}, \lambda_{4}\right)=\lambda_{3}$, the CSS and REE of $\rho_{B D}$ 


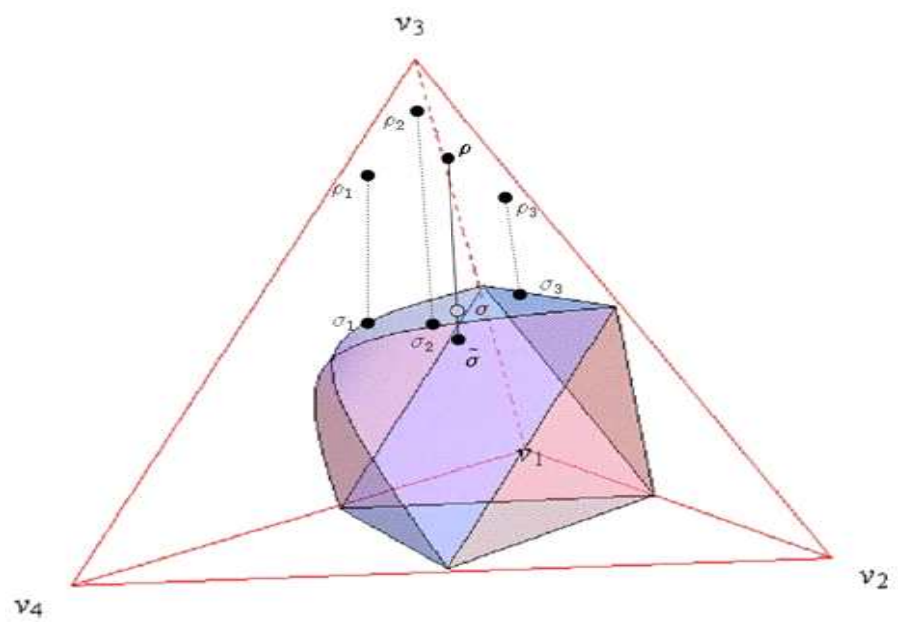

FIG. 1: (Color online) The schematic diagram of the procedure, by which REE can be computed from EOF. The polygon at the center is a deformed octahedron[12, 13. Inside and outside of the octahedron separable and entangled states reside, respectively. The CSS of the entangled state resides at the surface of the octahedron.

are

$$
\begin{gathered}
\pi_{B D}=\frac{\lambda_{1}}{2\left(1-\lambda_{3}\right)}\left|\beta_{1}\right\rangle\left\langle\beta_{1}\left|+\frac{\lambda_{2}}{2\left(1-\lambda_{3}\right)}\right| \beta_{2}\right\rangle\left\langle\beta_{2}\left|+\frac{1}{2}\right| \beta_{3}\right\rangle\left\langle\beta_{3}\left|+\frac{\lambda_{4}}{2\left(1-\lambda_{3}\right)}\right| \beta_{4}\right\rangle\left\langle\beta_{4}\right| \\
\mathcal{E}_{R}\left(\rho_{B D}\right)=-h\left(\lambda_{3}\right)+\ln 2 .
\end{gathered}
$$

Now, we will show that the procedure we suggested also yields the same result. Following Wootters procedure, one can show that the optimal decomposition of $\rho_{B D}$ for $\lambda_{3} \geq 1 / 2^{2}$ is

$$
\rho_{B D}=\sum_{j=0}^{4} p_{j}\left|\psi_{j}^{B D}\right\rangle\left\langle\psi_{j}^{B D}\right|
$$

where $p_{j}=1 / 4 \quad(j=1, \cdots, 4)$ and

$$
\begin{aligned}
& \left|\psi_{1}^{B D}\right\rangle=\sqrt{\lambda_{1}}\left|\beta_{1}\right\rangle+i \sqrt{\lambda_{2}}\left|\beta_{2}\right\rangle+\sqrt{\lambda_{3}}\left|\beta_{3}\right\rangle+\sqrt{\lambda_{4}}\left|\beta_{4}\right\rangle \\
& \left|\psi_{2}^{B D}\right\rangle=\sqrt{\lambda_{1}}\left|\beta_{1}\right\rangle+i \sqrt{\lambda_{2}}\left|\beta_{2}\right\rangle-\sqrt{\lambda_{3}}\left|\beta_{3}\right\rangle-\sqrt{\lambda_{4}}\left|\beta_{4}\right\rangle \\
& \left|\psi_{3}^{B D}\right\rangle=\sqrt{\lambda_{1}}\left|\beta_{1}\right\rangle-i \sqrt{\lambda_{2}}\left|\beta_{2}\right\rangle+\sqrt{\lambda_{3}}\left|\beta_{3}\right\rangle-\sqrt{\lambda_{4}}\left|\beta_{4}\right\rangle \\
& \left|\psi_{4}^{B D}\right\rangle=\sqrt{\lambda_{1}}\left|\beta_{1}\right\rangle-i \sqrt{\lambda_{2}}\left|\beta_{2}\right\rangle-\sqrt{\lambda_{3}}\left|\beta_{3}\right\rangle+\sqrt{\lambda_{4}}\left|\beta_{4}\right\rangle .
\end{aligned}
$$

${ }^{2}$ If $\lambda_{3} \leq 1 / 2, \rho_{B D}$ is a separable state. 
All $\left|\psi_{j}^{B D}\right\rangle \quad(j=1, \cdots, 4)$ have the same concurrence $\mathcal{C}=2 \lambda_{3}-1$ and, hence, the same $\lambda_{ \pm}$ (defined in Eq. A.2) as

$$
\lambda_{ \pm}=\frac{1}{2}\left(\sqrt{\lambda_{3}} \pm \sqrt{1-\lambda_{3}}\right)^{2} .
$$

The Schmidt bases of $\left|\psi_{1}^{B D}\right\rangle$ can be explicitly derived by following the procedure of appendix $\mathrm{A}$ and the result is

$$
\begin{aligned}
& \left|0_{A}\right\rangle=\frac{1}{N_{+}}\left[\left(\sqrt{1-\lambda_{3}}+\sqrt{\lambda_{4}}\right)|0\rangle+\left(\sqrt{\lambda_{1}}-i \sqrt{\lambda_{2}}\right)|1\rangle\right] \\
& \left|1_{A}\right\rangle=\frac{-1}{N_{-}}\left[\left(\sqrt{1-\lambda_{3}}-\sqrt{\lambda_{4}}\right)|0\rangle-\left(\sqrt{\lambda_{1}}-i \sqrt{\lambda_{2}}\right)|1\rangle\right] \\
& \left|0_{B}\right\rangle=\frac{1}{N_{+}}\left[\left(\sqrt{\lambda_{1}}+i \sqrt{\lambda_{2}}\right)|0\rangle+\left(\sqrt{1-\lambda_{3}}+\sqrt{\lambda_{4}}\right)|1\rangle\right] \\
& \left|1_{B}\right\rangle=\frac{1}{N_{-}}\left[\left(\sqrt{\lambda_{1}}+i \sqrt{\lambda_{2}}\right)|0\rangle-\left(\sqrt{1-\lambda_{3}}-\sqrt{\lambda_{4}}\right)|1\rangle\right],
\end{aligned}
$$

where the normalization constants $N_{ \pm}$are

$$
N_{ \pm}=\sqrt{2 \sqrt{1-\lambda_{3}}\left(\sqrt{1-\lambda_{3}} \pm \sqrt{\lambda_{4}}\right)}
$$

Thus the CSS of $\left|\psi_{1}^{B D}\right\rangle$, say $\sigma_{1}$, can be straightforwardly computed by making use of Eq. (A.6);

$$
\begin{aligned}
\sigma_{1} & =\lambda_{+}\left|0_{A} 0_{B}\right\rangle\left\langle 0_{A} 0_{B}\left|+\lambda_{-}\right| 1_{A} 1_{B}\right\rangle\left\langle 1_{A} 1_{B}\right| \\
& =\frac{1}{4\left(1-\lambda_{3}\right)}\left(\begin{array}{cccc}
\mu \mu^{*} & \mu \nu_{+} & \mu \nu_{-} & \mu^{2} \\
\mu^{*} \nu_{+} & d_{+} & \mu \mu^{*} & \mu \nu_{+} \\
\mu^{*} \nu_{-} & \mu \mu^{*} & d_{-} & \mu \nu_{-} \\
\left(\mu^{*}\right)^{2} & \mu^{*} \nu_{+} & \mu^{*} \nu_{-} & \mu \mu^{*}
\end{array}\right),
\end{aligned}
$$

where

$$
\begin{gathered}
\mu=\sqrt{\lambda_{1}}+i \sqrt{\lambda_{2}} \quad \nu_{ \pm}=2\left(1-\lambda_{3}\right) \sqrt{\lambda_{3}} \pm \sqrt{\lambda_{4}} \\
d_{ \pm}=\left(1-\lambda_{3}+\lambda_{4}\right) \pm 4\left(1-\lambda_{3}\right) \sqrt{\lambda_{3} \lambda_{4}} .
\end{gathered}
$$

Similarly, one can derive the Schmidt bases for other $\left|\psi_{j}^{B D}\right\rangle(j=2,3,4)$ and the corresponding $\operatorname{CSS} \sigma_{j}$. Then, one can show that the separable state $\tilde{\sigma}=\sum_{j=1}^{4} p_{j} \sigma_{j}$ with $p_{j}=1 / 4$ for all $j$ is

$$
\tilde{\sigma}=\frac{1}{4\left(1-\lambda_{3}\right)}\left(\begin{array}{cccc}
\lambda_{1}+\lambda_{2} & 0 & 0 & \lambda_{1}-\lambda_{2} \\
0 & 1-\lambda_{3}+\lambda_{4} & \lambda_{1}+\lambda_{2} & 0 \\
0 & \lambda_{1}+\lambda_{2} & 1-\lambda_{3}+\lambda_{4} & 0 \\
\lambda_{1}-\lambda_{2} & 0 & 0 & \lambda_{1}+\lambda_{2}
\end{array}\right) .
$$


This is a boundary state in the convex set of the separable states, because the minimal eigenvalue of its partial transposition, say $\tilde{\sigma}^{\Gamma}$, is zero. Thus, the procedure mentioned in the Introduction is terminated with identifying $\sigma_{*}=\tilde{\sigma}$. In fact, it is easy to show that $\tilde{\sigma}$ is exactly the same with $\pi_{B D}$ in Eq. (2.3). Thus, the procedure we suggested correctly derives the CSS of the Bell-diagonal states.

\section{GENERALIZED VEDRAL-PLENIO STATE}

In this section we will derive the CSS of the generalized Vedral-Plenio (GVP) state defined as

$$
\rho_{v p}=\lambda_{1}\left|\beta_{3}\right\rangle\left\langle\beta_{3}\left|+\lambda_{2}\right| 01\right\rangle\left\langle 01\left|+\lambda_{3}\right| 10\right\rangle\langle 10| \quad\left(\lambda_{1}+\lambda_{2}+\lambda_{3}=1\right)
$$

by following the procedure mentioned above. In fact the CSS and REE of the GVP were explicitly derived in Ref. [13] using a geometric argument, which are

$$
\begin{aligned}
& \pi_{v p}=\left(\frac{\lambda_{1}}{2}+\lambda_{2}\right)|01\rangle\left\langle 01\left|+\left(\frac{\lambda_{1}}{2}+\lambda_{3}\right)\right| 10\right\rangle\langle 10| \\
& \mathcal{E}_{R}\left(\rho_{v p}\right)=h\left(\frac{\lambda_{1}}{2}+\lambda_{2}\right)-h\left(\Lambda_{+}\right)
\end{aligned}
$$

where

$$
\Lambda_{ \pm}=\frac{1}{2}\left[1 \pm \sqrt{\lambda_{1}^{2}+\left(\lambda_{2}-\lambda_{3}\right)^{2}}\right]
$$

Now, we define

$$
a=\frac{\lambda_{1} \Lambda_{+}}{\sqrt{\lambda_{1}^{2}+\left(\lambda_{2}-\lambda_{3}\right)^{2}}} \quad b=-\frac{\left(\lambda_{2}-\lambda_{3}\right) \sqrt{\Lambda_{+} \Lambda_{-}}}{\sqrt{\lambda_{1}^{2}+\left(\lambda_{2}-\lambda_{3}\right)^{2}}} \quad c=-\frac{\lambda_{1} \Lambda_{-}}{\sqrt{\lambda_{1}^{2}+\left(\lambda_{2}-\lambda_{3}\right)^{2}}}
$$

and $\Omega^{2}=2\left[(a-c)^{2}+4 b^{2}-(a-c) \sqrt{(a-c)^{2}+4 b^{2}}\right]$. We also define the unnormalized states $\left|v_{ \pm}\right\rangle=\sqrt{\Lambda_{ \pm}}\left|\Lambda_{ \pm}\right\rangle$, where $\left|\Lambda_{ \pm}\right\rangle$are eigenstates of $\rho_{v p}$

$$
\begin{aligned}
& \left|\Lambda_{+}\right\rangle=\frac{1}{N}\left[\left(\sqrt{\lambda_{1}^{2}+\left(\lambda_{2}-\lambda_{3}\right)^{2}}+\left(\lambda_{2}-\lambda_{3}\right)\right)|01\rangle+\lambda_{1}|10\rangle\right] \\
& \left|\Lambda_{-}\right\rangle=\frac{1}{N}\left[\lambda_{1}|01\rangle-\left(\sqrt{\lambda_{1}^{2}+\left(\lambda_{2}-\lambda_{3}\right)^{2}}+\left(\lambda_{2}-\lambda_{3}\right)\right)|10\rangle\right] .
\end{aligned}
$$

In Eq. (3.5) $N$ is a normalization constant given by

$$
N^{2}=2 \sqrt{\lambda_{1}^{2}+\left(\lambda_{2}-\lambda_{3}\right)^{2}}\left\{\sqrt{\lambda_{1}^{2}+\left(\lambda_{2}-\lambda_{3}\right)^{2}}+\left(\lambda_{2}-\lambda_{3}\right)\right\} .
$$


Then, following Ref.[7], the optimal decomposition of $\rho_{v p}$ for EOF is $\rho_{v p}=$ $\sum_{j=1}^{2} p_{j}\left|\psi_{j}^{V P}\right\rangle\left\langle\psi_{j}^{V P}\right|$, where $p_{1}=p_{2}=1 / 2$ and

$$
\begin{aligned}
& \left|\psi_{1}^{V P}\right\rangle=\frac{-i}{\Omega}\left[2 b-i\left\{\sqrt{(a-c)^{2}+4 b^{2}}-(a-c)\right\}\right]\left(\left|v_{+}\right\rangle+i\left|v_{-}\right\rangle\right) \\
& \left|\psi_{2}^{V P}\right\rangle=\frac{-i}{\Omega}\left[2 b+i\left\{\sqrt{(a-c)^{2}+4 b^{2}}-(a-c)\right\}\right]\left(\left|v_{+}\right\rangle-i\left|v_{-}\right\rangle\right) .
\end{aligned}
$$

Following appendix A one can derive the CSS for $\left|\psi_{j}^{V P}\right\rangle$ directly. Then, one can realize that $\left|\psi_{1}^{V P}\right\rangle$ and $\left|\psi_{2}^{V P}\right\rangle$ have the same CSS, which is identical with $\pi_{v p}$. Thus, the procedure also gives a correct CSS for the GVP states.

\section{GENERALIZED HORODECKI STATES}

In this section we will show that the procedure also generates the correct CSS for the generalized Horodecki states

$$
\rho_{H}=\lambda_{1}\left|\beta_{3}\right\rangle\left\langle\beta_{3}\left|+\lambda_{2}\right| 00\right\rangle\left\langle 00\left|+\lambda_{3}\right| 11\right\rangle\langle 11|
$$

with $\lambda_{1}+\lambda_{2}+\lambda_{3}=1$ and $\lambda_{1} \geq 2{\sqrt{\lambda_{2} \lambda_{3}}}^{3}$. The CSS and REE of $\rho_{H}$ were derived in Ref. [13] using a geometrical argument and the results are

$$
\begin{aligned}
& \pi_{H}=\frac{\left(\lambda_{1}+2 \lambda_{2}\right)\left(\lambda_{1}+2 \lambda_{3}\right)}{2}\left|\beta_{3}\right\rangle\left\langle\beta_{3}\left|+\frac{\left(\lambda_{1}+2 \lambda_{2}\right)^{2}}{4}\right| 00\right\rangle\left\langle 00\left|+\frac{\left(\lambda_{1}+2 \lambda_{3}\right)^{2}}{4}\right| 11\right\rangle\langle 11| \\
& \mathcal{E}_{R}\left(\rho_{H}\right)=\lambda_{1} \ln \lambda_{1}+\lambda_{2} \ln \lambda_{2}+\lambda_{3} \ln \lambda_{3}+2 h\left(\frac{\lambda_{1}}{2}+\lambda_{2}\right)-\lambda_{1} \ln 2 .
\end{aligned}
$$

Following Ref.[7] one can straightforwardly construct the optimal decomposition of $\rho_{H}$ for EOF, which is $\rho_{H}=\sum_{j=1}^{3} p_{j}\left|\psi_{j}^{H}\right\rangle\left\langle\psi_{j}^{H}\right|$, where $p_{1}=p_{2}=p_{3}=1 / 3$ and

$$
\begin{aligned}
& \left|\psi_{1}^{H}\right\rangle=\sqrt{\lambda_{1}}\left|\beta_{3}\right\rangle+\sqrt{\lambda_{2}}|00\rangle+\sqrt{\lambda_{3}}|11\rangle \\
& \left|\psi_{2}^{H}\right\rangle=\sqrt{\lambda_{1}}\left|\beta_{3}\right\rangle+\sqrt{\lambda_{2}} e^{i 2 \pi / 3}|00\rangle+\sqrt{\lambda_{3}} e^{-i 2 \pi / 3}|11\rangle \\
& \left|\psi_{3}^{H}\right\rangle=\sqrt{\lambda_{1}}\left|\beta_{3}\right\rangle+\sqrt{\lambda_{2}} e^{i 4 \pi / 3}|00\rangle+\sqrt{\lambda_{3}} e^{-i 4 \pi / 3}|11\rangle .
\end{aligned}
$$

In order to treat $\left|\psi_{j}^{H}\right\rangle$ as an unified manner let us consider $|\phi\rangle=\sqrt{\lambda_{1}}\left|\beta_{3}\right\rangle+\sqrt{\lambda_{2}} e^{i \theta}|00\rangle+$ $\sqrt{\lambda_{3}} e^{-i \theta}|11\rangle$. Then, $\lambda_{ \pm}$defined in Eq. A.2 is

$$
\lambda_{ \pm}=\left(\frac{R \pm\left(\sqrt{\lambda_{2}}+\sqrt{\lambda_{3}}\right)}{2}\right)^{2}
$$

\footnotetext{
${ }^{3}$ If $\lambda_{1} \leq 2 \sqrt{\lambda_{2} \lambda_{3}}, \rho_{H}$ becomes a separable state.
} 
where $R=\sqrt{2 \lambda_{1}+\left(\sqrt{\lambda_{2}}-\sqrt{\lambda_{3}}\right)^{2}}$. Since $\lambda_{ \pm}$is independent of $\theta$, this fact indicates that $\lambda_{ \pm}$ of $\left|\psi_{j}^{H}\right\rangle$ are equal to Eq. 4.4 for all $j$. Following appendix A, it is straightforward to show that the Schmidt bases of $|\phi\rangle$ are

$$
\begin{gathered}
\left|0_{A}\right\rangle=\sqrt{\frac{\lambda_{1}}{R\left[R-\left(\sqrt{\lambda_{2}}-\sqrt{\lambda_{3}}\right)\right]}}|0\rangle+\sqrt{\frac{R-\left(\sqrt{\lambda_{2}}-\sqrt{\lambda_{3}}\right)}{2 R}} e^{-i \theta}|1\rangle \\
\left|1_{A}\right\rangle=-\sqrt{\frac{\lambda_{1}}{R\left[R+\left(\sqrt{\lambda_{2}}-\sqrt{\lambda_{3}}\right)\right]}}|0\rangle+\sqrt{\frac{R+\left(\sqrt{\lambda_{2}}-\sqrt{\lambda_{3}}\right)}{2 R}} e^{-i \theta}|1\rangle \\
\left|0_{B}\right\rangle=e^{i \theta}\left|0_{A}\right\rangle \quad\left|1_{B}\right\rangle=-e^{i \theta}\left|1_{A}\right\rangle .
\end{gathered}
$$

Then the CSS $\sigma_{\phi}$ of $|\phi\rangle$ is

$$
\begin{aligned}
\sigma_{\phi} \equiv & \lambda_{+}\left|0_{A} 0_{B}\right\rangle\left\langle 0_{A} 0_{B}\left|+\lambda_{-}\right| 1_{A} 1_{B}\right\rangle\left\langle 1_{A} 1_{B}\right| \\
= & \left(\begin{array}{cccc}
\frac{\lambda_{1}+2 \lambda_{2}}{2}-\frac{\lambda_{1}}{2 R^{2}} & \mathcal{A} e^{i \theta} & \mathcal{A} e^{i \theta} & \frac{\lambda_{1}}{2 R^{2}} e^{2 i \theta} \\
\mathcal{A} e^{-i \theta} & \frac{\lambda_{1}}{2 R^{2}} & \frac{\lambda_{1}}{2 R^{2}} & \mathcal{B} e^{i \theta} \\
\mathcal{A} e^{-i \theta} & \frac{\lambda_{1}}{2 R^{2}} & \frac{\lambda_{1}}{2 R^{2}} & \mathcal{B} e^{i \theta} \\
\frac{\lambda_{1}}{2 R^{2}} e^{-2 i \theta} & \mathcal{B} e^{-i \theta} & \mathcal{B} e^{-i \theta} & \frac{\lambda_{1}+2 \lambda_{3}}{2}-\frac{\lambda_{1}}{2 R^{2}}
\end{array}\right)
\end{aligned}
$$

where

$$
\begin{aligned}
& \mathcal{A}=\frac{\sqrt{2 \lambda_{1}}}{4 R^{2}}\left[2 \sqrt{\lambda_{2}}+\left(\sqrt{\lambda_{2}}+\sqrt{\lambda_{3}}\right)\left(\lambda_{1}-2 \sqrt{\lambda_{2} \lambda_{3}}\right)\right] \\
& \mathcal{B}=\frac{\sqrt{2 \lambda_{1}}}{4 R^{2}}\left[2 \sqrt{\lambda_{3}}+\left(\sqrt{\lambda_{2}}+\sqrt{\lambda_{3}}\right)\left(\lambda_{1}-2 \sqrt{\lambda_{2} \lambda_{3}}\right)\right] .
\end{aligned}
$$

Thus, the CSS $\sigma_{j}$ of $\left|\psi_{j}^{H}\right\rangle$ can be obtained by letting $\theta=0,2 \pi / 3,4 \pi / 3$, respectively.

Then, $\tilde{\sigma}=\sum_{j=1}^{3} p_{j} \sigma_{j}$ with $p_{j}=1 / 3(j=1,2,3)$ reduces

$$
\tilde{\sigma}=\left(\begin{array}{cccc}
\frac{\lambda_{1}+2 \lambda_{2}}{2}-\frac{\lambda_{1}}{2 R^{2}} & 0 & 0 & 0 \\
0 & \frac{\lambda_{1}}{2 R^{2}} & \frac{\lambda_{1}}{2 R^{2}} & 0 \\
0 & \frac{\lambda_{1}}{2 R^{2}} & \frac{\lambda_{1}}{2 R^{2}} & 0 \\
0 & 0 & 0 & \frac{\lambda_{1}+2 \lambda_{3}}{2}-\frac{\lambda_{1}}{2 R^{2}}
\end{array}\right) .
$$

However, $\tilde{\sigma}$ is not a boundary state in the convex set of the separable states, because the minimum eigenvalue of $\tilde{\sigma}^{\Gamma}$ is positive. Thus, we define

$$
\sigma_{*}=x \tilde{\sigma}+(1-x) \rho_{H} . \quad(0 \leq x \leq 1)
$$


The condition that the minimum eigenvalue of $\sigma_{*}^{\Gamma}$ is zero fixes $x$ as

$$
x=\frac{R^{2}}{2 \lambda_{1}}\left(\lambda_{1}+2 \sqrt{\lambda_{2} \lambda_{3}}\right) .
$$

Inserting Eq.4.10 into $\sigma_{*}$, one can show that $\sigma_{*}$ reduces to $\pi_{H}$. Thus, our procedure gives a correct CSS for the generalized Horodecki states.

\section{LESS SYMMETRIC STATES}

In the previous sections we have shown that the procedure generates the correct CSS and REE for various symmetric states such as Bell-diagonal, GVP, and generalized Horodecki states. In this section we will apply the procedure to the less symmetric states.

\section{A. Vedral-Plenio-Type State}

The first quantum state we consider is

$$
\Sigma_{1}=A_{2}|01\rangle\left\langle 01\left|+A_{3}\right| 10\right\rangle\langle 10|+D(|01\rangle\langle 10|+| 10\rangle\langle 01|),
$$

where $A_{2}+A_{3}=1, A_{2} \geq A_{3}$ and $0 \leq D \leq \sqrt{A_{2} A_{3}}$. Of course, if $A_{2}=\frac{\lambda_{1}}{2}+\lambda_{2}, A_{3}=\frac{\lambda_{1}}{2}+\lambda_{3}$, and $D=\frac{\lambda_{1}}{2}, \Sigma_{1}$ reduces to $\rho_{v p}$ in Eq. (3.1). Thus, we call $\Sigma_{1}$ as Vedral-Plenio-type state.

In order to apply the procedure to $\Sigma_{1}$ we introduce

$$
\begin{array}{ll}
R=\sqrt{\left(A_{2}-A_{3}\right)^{2}+4 D^{2}} & \tan 2 \theta=\frac{2 D}{A_{2}-A_{3}} \\
\lambda_{1}=\frac{1}{2}\left[\left(A_{2}+A_{3}\right)+R\right] & \lambda_{2}=\frac{1}{2}\left[\left(A_{2}+A_{3}\right)-R\right] \\
\left|\lambda_{1}\right\rangle=\cos \theta|01\rangle+\sin \theta|10\rangle & \left|\lambda_{2}\right\rangle=\sin \theta|01\rangle-\cos \theta|10\rangle .
\end{array}
$$

Applying Ref.[7], it is possible to derive the optimal decomposition of $\Sigma_{1}$ for EOF; $\Sigma_{1}=$ $p_{1}\left|w_{1}\right\rangle\left\langle w_{1}\left|+p_{2}\right| w_{2}\right\rangle\left\langle w_{2}\right|$, where

$$
p_{1}=\frac{1}{2}\left[1+\frac{A_{2}-A_{3}}{\sqrt{1-4 D^{2}}}\right] \quad p_{2}=\frac{1}{2}\left[1-\frac{A_{2}-A_{3}}{\sqrt{1-4 D^{2}}}\right]
$$

and

$$
\begin{aligned}
& \left|w_{1}\right\rangle=\frac{1}{\mathcal{Y}_{+}}\left[\left(\sqrt{\xi_{+} \eta_{+}}+\sqrt{\xi_{-} \eta_{-}}\right) \sqrt{\lambda_{1}}\left|\lambda_{1}\right\rangle+\left(\sqrt{\xi_{+} \eta_{-}}-\sqrt{\xi_{-} \eta_{+}}\right) \sqrt{\lambda_{2}}\left|\lambda_{2}\right\rangle\right] \\
& \left|w_{2}\right\rangle=\frac{1}{\mathcal{Y}_{-}}\left[\left(\sqrt{\xi_{+} \eta_{-}}-\sqrt{\xi_{-} \eta_{+}}\right) \sqrt{\lambda_{1}}\left|\lambda_{1}\right\rangle-\left(\sqrt{\xi_{+} \eta_{+}}+\sqrt{\xi_{-} \eta_{-}}\right) \sqrt{\lambda_{2}}\left|\lambda_{2}\right\rangle\right] .
\end{aligned}
$$


In Eq. (5.4) $\xi_{ \pm}, \eta_{ \pm}$, and $\mathcal{Y}_{ \pm}$are

$$
\begin{gathered}
\xi_{ \pm}=R \sqrt{A_{2} A_{3}} \pm D\left(A_{2}+A_{3}\right) \quad \eta_{ \pm}=\sqrt{A_{2} A_{3}\left(1-4 D^{2}\right)} \pm D\left(A_{2}-A_{3}\right) \\
\mathcal{Y}_{ \pm}^{2}=2 A_{2} A_{3} R\left[\sqrt{1-4 D^{2}} \pm\left(A_{2}-A_{3}\right)\right]
\end{gathered}
$$

Following appendix A, one can derive the CSS $\sigma_{1}$ and $\sigma_{2}$ of $\left|w_{1}\right\rangle$ and $\left|w_{2}\right\rangle$ after long and tedious calculation. The final results are

$$
\begin{aligned}
\sigma_{1} & =\left[\frac{\cos \theta \sqrt{\lambda_{1}}\left(\sqrt{\xi_{+} \eta_{+}}+\sqrt{\xi_{-} \eta_{-}}\right)+\sin \theta \sqrt{\lambda_{2}}\left(\sqrt{\xi_{+} \eta_{-}}-\sqrt{\xi_{-} \eta_{+}}\right)}{\mathcal{Y}_{+}}\right]^{2}|01\rangle\langle 01| \\
+ & {\left[\frac{\sin \theta \sqrt{\lambda_{1}}\left(\sqrt{\xi_{+} \eta_{+}}+\sqrt{\xi_{-} \eta_{-}}\right)-\cos \theta \sqrt{\lambda_{2}}\left(\sqrt{\xi_{+} \eta_{-}}-\sqrt{\xi_{-} \eta_{+}}\right)}{\mathcal{Y}_{+}}\right]^{2}|10\rangle\langle 10| } \\
\sigma_{2} & =\left[\frac{\cos \theta \sqrt{\lambda_{1}}\left(\sqrt{\xi_{+} \eta_{-}}-\sqrt{\xi_{-} \eta_{+}}\right)-\sin \theta \sqrt{\lambda_{2}}\left(\sqrt{\xi_{+} \eta_{+}}+\sqrt{\xi_{-} \eta_{-}}\right)}{\mathcal{Y}_{-}}\right]^{2}|01\rangle\langle 01| \\
& +\left[\frac{\sin \theta \sqrt{\lambda_{1}}\left(\sqrt{\xi_{+} \eta_{-}}-\sqrt{\xi_{-} \eta_{+}}\right)+\cos \theta \sqrt{\lambda_{2}}\left(\sqrt{\xi_{+} \eta_{+}}+\sqrt{\xi_{-} \eta_{-}}\right)}{\mathcal{Y}_{-}}\right]^{2}|10\rangle\langle 10| .
\end{aligned}
$$

Then, $\tilde{\sigma}=p_{1} \sigma_{1}+p_{2} \sigma_{2}$ simply reduces to

$$
\tilde{\sigma}=A_{2}|01\rangle\left\langle 01\left|+A_{3}\right| 10\right\rangle|10\rangle
$$

This is manifestly boundary state in the convex set of separable states. Thus, the procedure states that $\tilde{\sigma}$ is a CSS of $\Sigma_{1}$. This is exactly the same with theorem 1 of Ref. [14].

\section{B. Horodecki-Type State}

The second less symmetric quantum state we consider is

$$
\Sigma_{2}=\left(\begin{array}{cccc}
A_{1} & 0 & 0 & 0 \\
0 & A & D & 0 \\
0 & D & A & 0 \\
0 & 0 & 0 & A_{4}
\end{array}\right)
$$

where $A_{1}+A_{4}+2 A=1$ and $\sqrt{A_{1} A_{4}}<D \leq A$. If $A=D=\lambda_{1} / 2, A_{1}=\lambda_{2}$, and $A_{4}=\lambda_{3}, \Sigma_{2}$ reduces to $\rho_{H}$ in Eq. (4.1). Thus, we call $\Sigma_{2}$ as Horodecki-type state. Applying Ref.[7], one 
can derive the optimal decomposition of $\Sigma_{2}$ for EOF as $\Sigma_{2}=\sum_{j=1}^{4} p_{j}\left|h_{j}\right\rangle$, where $p_{j}=1 / 4$ for all $j$ and

$$
\begin{aligned}
& \left|h_{1}\right\rangle=\sqrt{A+D}\left|\beta_{3}\right\rangle+\sqrt{A-D}\left|\beta_{4}\right\rangle+\sqrt{A_{1}}|00\rangle+\sqrt{A_{4}}|11\rangle \\
& \left|h_{2}\right\rangle=\sqrt{A+D}\left|\beta_{3}\right\rangle+\sqrt{A-D}\left|\beta_{4}\right\rangle-\sqrt{A_{1}}|00\rangle-\sqrt{A_{4}}|11\rangle \\
& \left|h_{3}\right\rangle=\sqrt{A+D}\left|\beta_{3}\right\rangle-\sqrt{A-D}\left|\beta_{4}\right\rangle+i \sqrt{A_{1}}|00\rangle-i \sqrt{A_{4}}|11\rangle \\
& \left|h_{4}\right\rangle=\sqrt{A+D}\left|\beta_{3}\right\rangle-\sqrt{A-D}\left|\beta_{4}\right\rangle-i \sqrt{A_{1}}|00\rangle+i \sqrt{A_{4}}|11\rangle .
\end{aligned}
$$

In order to consider $\left|h_{j}\right\rangle(j=1, \cdots, 4)$ all together, we define

$$
\begin{aligned}
& \left|\varphi_{1}\right\rangle=\sqrt{A+D}\left|\beta_{3}\right\rangle+\sqrt{A-D}\left|\beta_{4}\right\rangle+e^{i \theta} \sqrt{A_{1}}|00\rangle+e^{-i \theta} \sqrt{A_{4}}|11\rangle \\
& \left|\varphi_{2}\right\rangle=\sqrt{A+D}\left|\beta_{3}\right\rangle-\sqrt{A-D}\left|\beta_{4}\right\rangle+e^{i \theta} \sqrt{A_{1}}|00\rangle+e^{-i \theta} \sqrt{A_{4}}|11\rangle .
\end{aligned}
$$

For $\left|\varphi_{1}\right\rangle$ the Schmidt bases are

$$
\begin{aligned}
&\left|0_{A}\right\rangle=\frac{1}{2 \mathcal{Z}_{+}} {[\sqrt{2}(\sqrt{A-D} \sqrt{1+\mathcal{C}}+\sqrt{A+D} \sqrt{1-\mathcal{C}})|0\rangle} \\
&+\left.+e^{-i \theta}\left\{\left(\sqrt{A_{1}}+\sqrt{A_{4}}\right) \sqrt{1+\mathcal{C}}-\left(\sqrt{A_{1}}-\sqrt{A_{4}}\right) \sqrt{1-\mathcal{C}}\right\}|1\rangle\right] \\
&\left|1_{A}\right\rangle=\frac{1}{2 \mathcal{Z}_{-}} {[\sqrt{2}(\sqrt{A-D} \sqrt{1+\mathcal{C}}-\sqrt{A+D} \sqrt{1-\mathcal{C}})|0\rangle} \\
&\left.+e^{-i \theta}\left\{\left(\sqrt{A_{1}}+\sqrt{A_{4}}\right) \sqrt{1+\mathcal{C}}+\left(\sqrt{A_{1}}-\sqrt{A_{4}}\right) \sqrt{1-\mathcal{C}}\right\}|1\rangle\right] \\
&\left|0_{B}\right\rangle=\frac{1}{2 \mathcal{Z}_{+}}\left[\sqrt{2} e^{i \theta}\left\{\sqrt{A+D}\left(\sqrt{A_{1}}+\sqrt{A_{4}}\right)+\sqrt{A-D}\left(\sqrt{A_{1}}-\sqrt{A_{4}}\right)\right\}|0\rangle\right. \\
&\left.+\left\{-\left(A_{1}-A_{4}\right)+2 \sqrt{A^{2}-D^{2}}+\sqrt{1-\mathcal{C}^{2}}\right\}|1\rangle\right] \\
&\left|1_{B}\right\rangle=\frac{1}{2 \mathcal{Z}_{-}}\left[\sqrt{2} e^{i \theta}\left\{\sqrt{A+D}\left(\sqrt{A_{1}}+\sqrt{A_{4}}\right)+\sqrt{A-D}\left(\sqrt{A_{1}}-\sqrt{A_{4}}\right)\right\}|0\rangle\right. \\
&\left.+\left\{-\left(A_{1}-A_{4}\right)+2 \sqrt{A^{2}-D^{2}}-\sqrt{1-\mathcal{C}^{2}}\right\}|1\rangle\right],
\end{aligned}
$$

where $\mathcal{C}=2\left(D-\sqrt{A_{1} A_{4}}\right)$ and

$$
\mathcal{Z}_{ \pm}^{2}=\frac{1}{2} \sqrt{1-\mathcal{C}^{2}}\left[\sqrt{1-\mathcal{C}^{2}} \mp\left(A_{1}-A_{4}\right) \pm 2 \sqrt{A^{2}-D^{2}}\right] .
$$

Thus, the $\operatorname{CSS} \sigma_{1}(\theta)$ of $\left|\varphi_{1}\right\rangle$ is

$$
\sigma_{1}(\theta)=\left(\frac{\sqrt{1+\mathcal{C}}+\sqrt{1-\mathcal{C}}}{2}\right)^{2}\left|0_{A} 0_{B}\right\rangle\left\langle 0_{A} 0_{B}\left|+\left(\frac{\sqrt{1+\mathcal{C}}-\sqrt{1-\mathcal{C}}}{2}\right)^{2}\right| 1_{A} 1_{B}\right\rangle\left\langle 1_{A} 1_{B}\right| .
$$


Similarly, it is straightforward to derive the $\operatorname{CSS} \sigma_{2}(\theta)$ of $\left|\varphi_{2}\right\rangle$. Then, one can show

$$
\begin{gathered}
\tilde{\Pi} \equiv \frac{1}{4}\left[\sigma_{1}(0)+\sigma_{1}(\pi)+\sigma_{2}\left(\frac{\pi}{2}\right)+\sigma_{2}\left(-\frac{\pi}{2}\right)\right] \\
=\left(\begin{array}{cccc}
a_{1} & 0 & 0 & 0 \\
0 & a & d & 0 \\
0 & d & a & 0 \\
0 & 0 & 0 & a_{4}
\end{array}\right)
\end{gathered}
$$

where

$$
\begin{aligned}
& a_{1}=\frac{1}{4\left(1-\mathcal{C}^{2}\right)}\left[(1+\mathcal{C})\left(\sqrt{A_{1}}+\sqrt{A_{4}}\right)^{2}+(1-\mathcal{C})\left(\sqrt{A_{1}}-\sqrt{A_{4}}\right)^{2}\right. \\
& \left.+2\left(1-\mathcal{C}^{2}\right)\left(A_{1}-A_{4}\right)\right] \\
& a_{4}=\frac{1}{4\left(1-\mathcal{C}^{2}\right)}\left[(1+\mathcal{C})\left(\sqrt{A_{1}}+\sqrt{A_{4}}\right)^{2}+(1-\mathcal{C})\left(\sqrt{A_{1}}-\sqrt{A_{4}}\right)^{2}\right. \\
& \left.-2\left(1-\mathcal{C}^{2}\right)\left(A_{1}-A_{4}\right)\right] \\
& a=\frac{1}{2\left(1-\mathcal{C}^{2}\right)}[(1+\mathcal{C})(A-D)+(1-\mathcal{C})(A+D)] \\
& d=\frac{2 A \sqrt{A_{1} A_{4}}+D\left(A_{1}+A_{4}\right)}{1-\mathcal{C}^{2}} .
\end{aligned}
$$

One can show that if $A=D=\lambda_{1} / 2, A_{1}=\lambda_{2}$, and $A_{4}=\lambda_{3}$, $\tilde{\Pi}$ reduces to Eq. 4.8.

Since $\tilde{\Pi}$ is not a boundary state in the set of separable states, we define

$$
\Pi_{*}=x \tilde{\Pi}+(1-x) \Sigma_{2} .
$$

Then, the CSS condition of $\Pi_{*}$ is

$$
\left[x\left(a_{1}-A_{1}\right)+A_{1}\right]\left[x\left(a_{4}-A_{4}\right)+A_{4}\right]=[x(d-D)+D]^{2} .
$$

In the Horodecki state limit Eq. (5.17) gives a solution 4.10). Using $a_{1}-A_{1}=a_{4}-A_{4}=$ $-(a-A)=f /\left(1-\mathcal{C}^{2}\right)$ and $d-D=g /\left(1-\mathcal{C}^{2}\right)$ where

$$
f=\mathcal{C}(D-A \mathcal{C}) \quad g=\mathcal{C}(\mathcal{C} D-A)
$$

the solution of $x$, say $x=x_{*}$, can be obtained by solving the quadratic equation (5.17). Inserting $x=x_{*}$ in Eq. (5.16), one can compute $\Pi_{*}$ explicitly, which is a candidate of CSS for $\Sigma_{2}$. 
The CSS of $\Sigma_{2}$ was derived in the theorem 2 of Ref.[14] by using the converse procedure introduced in Ref.[16]. The explicit form of the CSS is

$$
\pi_{\Sigma_{2}}=\left(\begin{array}{cccc}
r_{1} & 0 & 0 & 0 \\
0 & r & y & 0 \\
0 & y & r & 0 \\
0 & 0 & 0 & r_{4}
\end{array}\right)
$$

where

$$
\begin{aligned}
& r_{1}=\frac{1}{F}\left[2 A_{1}\left(A_{1}+A_{2}\right)\left(A_{1}+A_{2}+A_{4}\right)-D^{2}\left(A_{1}-A_{4}\right)+\Delta\right] \\
& r_{4}=\frac{1}{F}\left[2 A_{4}\left(A_{2}+A_{4}\right)\left(A_{1}+A_{2}+A_{4}\right)+D^{2}\left(A_{1}-A_{4}\right)+\Delta\right] \\
& r=\frac{1}{F}\left[2\left(A_{1}+A_{2}\right)\left(A_{2}+A_{4}\right)\left(A_{1}+A_{2}+A_{4}\right)-D^{2}\left(A_{1}+2 A_{2}+A_{4}\right)-\Delta\right]
\end{aligned}
$$

and $y=\sqrt{r_{1} r_{4}}$. In Eq. (5.20) $D$ and $\Delta$ are

$$
\begin{aligned}
& F=2\left(A_{1}+A_{2}+A_{4}+D\right)\left(A_{1}+A_{2}+A_{4}-D\right) \\
& \Delta=D \sqrt{D^{2}\left(A_{1}-A_{4}\right)^{2}+4 A_{1} A_{4}\left(A_{1}+A_{2}\right)\left(A_{2}+A_{4}\right)} .
\end{aligned}
$$

Our candidate $\left.\Pi_{*}\right|_{x=x_{*}}$ does not coincide with the correct CSS $\pi_{\Sigma_{2}}$. Thus, the procedure does not give a correct REE for $\Sigma_{2}$, although it gives correct REE for Bell-diagonal, GVP, generalized Horodecki, and Vedral-Plenio-type states.

\section{CONCLUSION}

In this paper we examine the possibility for deriving the closed formula for REE in twoqubit system without relying on the converse procedure discussed in Ref. [16-18]. Since REE and EOF are identical for all pure states in spite of their different definitions, we think they should have some connection somehow. In this context we suggest a procedure, where REE can be computed from EOF. The procedure gives correct REE for many symmetric states such as Bell-diagonal, GVP, and generalized Horodecki states. It also generates a correct REE for less symmetric states such as $\Sigma_{1}$. However, the procedure failed to produce a correct REE for the less symmetric states $\Sigma_{2}$. This means our procedure is still incomplete for deriving the closed formula of REE. 
We think still the connection between EOF and REE is not fully revealed. If this connection is sufficiently understood in the future, probably the closed formula for REE can be derived. We hope to explore this issue in the future.

Acknowledgement: This research was supported by the Basic Science Research Program through the National Research Foundation of Korea(NRF) funded by the Ministry of Education, Science and Technology(2011-0011971).

[1] C. H. Bennett, D. P. DiVincenzo, J. A. Smokin and W. K. Wootters, Mixed-state entanglement and quantum error correction, Phys. Rev. A 54 (1996) 3824 quant-ph/9604024.

[2] V. Vedral, M. B. Plenio, M. A. Rippin and P. L. Knight, Quantifying Entanglement, Phys. Rev. Lett. 78 (1997) 2275 quant-ph/9702027.

[3] V. Vedral and M. B. Plenio, Entanglement measures and purification procedures, Phys. Rev. A 57 (1998) 1619 quant-ph/9707035.

[4] A. Shimony, Degree of entanglement, in D. M. Greenberg and A. Zeilinger (eds.), Fundamental problems in quantum theory: A conference held in honor of J. A. Wheeler, Ann. N. Y. Acad. Sci. 755 (1995) 675; H. Barnum and N. Linden, Monotones and Invariants for Multi-particle Quantum States, J. Phys. A: Math. Gen. 34, (2001) 6787 quant-ph/0103155]; T.-C. Wei and P. M. Goldbart, Geometric measure of entanglement and application to bipartite and multipartite quantum states, Phys. Rev. A 68 (2003) 042307 quant-ph/0307219].

[5] A. Uhlmann, Fidelity and concurrence of conjugate states, Phys. Rev. A 62 (2000) 032307 quant-ph/9909060.

[6] S. Hill and W. K. Wootters, Entanglement of a pair of quantum bits, Phys. Rev. Lett. 78 (1997) 5022 quant-ph/9703041.

[7] W. K. Wootters, Entanglement of Formation of an Arbitrary State of Two Qubits, Phys. Rev. Lett. 80 (1998) 2245 quant-ph/9709029.

[8] V. Coffman, J. Kundu and W. K. Wootters, Distributed entanglement, Phys. Rev. A 61 (2000) 052306 quant-ph/9907047.

[9] A. Osterloh and J. Siewert, Constructing N-qubit entanglement monotones from antilinear operators, Phys. Rev. A 72 (2005) 012337 quant-ph/0410102]; D. Ž. Doković and A. Osterloh, On polynomial invariants of several qubits, J. Math. Phys. 50 (2009) 033509 arXiv:0804.1661 
(quant-ph)].

[10] M. Sarovar, A. Ishizaki, G. R. Fleming, K. B. Whaley, Quantum entanglement in photosynthetic light harvesting complexes, Nature Physics, 6(2010) 462 arXiv:0905.3787 (quant-ph)] and references therein.

[11] O. Krueger and R. F. Werner, Some Open Problems in Quantum Information Theory, quant$\mathrm{ph} / 0504166$.

[12] R. Horodecki and M. Horodecki, Information-theoretic aspects of inseparability of mixed states, Phys. Rev. A 54, (1996) 1838 quant-ph/9607007].

[13] H. Kim, M. R. Hwang, E. Jung and D. K. Park, Difficulties in analytic computation for relative entropy of entanglement, Phys. Rev. A 81 (2010) 052325 [arXiv:1002.4695 (quant-ph)].

[14] D. K. Park, Relative entropy of entanglement for two-qubit state with $z$-directional Bloch vectors, Int. J. Quant. Inf. 8 (2010) 869 [arXiv:1005.4777 (quant-ph)].

[15] M. Horodecki, P. Horodecki, and R. Horodecki, in Quantum Information: An Introduction to Basic Theoretical Concepts and Experiments, edited by G. Alber et al. (Springer, Berlin, 2001), p. 151.

[16] A. Miranowicz and S. Ishizaka, Closed formula for the relative entropy of entanglement, Phys. Rev. A78 (2008) 032310 [arXiv:0805.3134 (quant-ph)].

[17] S. Friedland and G Gour, Closed formula for the relative entropy of entanglement in all dimensions, J. Math. Phys. 52 (2011) 052201 arXiv:1007.4544 (quant-ph)].

[18] M. W. Girard, G. Gour, and S. Friedland, On convex optimization problems in quantum information theory, arXiv:1402.0034 (quant-ph). 


\section{Appendix A}

In this section we will show that REE and EOF are identical for two-qubit pure states. This fact was already proven in Theorem 3 of Ref. [3]. We will prove this again more directly, because explicit Schmidt bases are used in the main body of the paper.

Let us consider a general two-qubit pure state $\left|\psi_{2}\right\rangle_{A B}=\alpha_{1}|00\rangle+\alpha_{2}|01\rangle+\alpha_{3}|10\rangle+\alpha_{4}|11\rangle$ with $\left|\alpha_{1}\right|^{2}+\left|\alpha_{2}\right|^{2}+\left|\alpha_{3}\right|^{2}+\left|\alpha_{4}\right|^{2}=1$. Then, its concurrence is $\mathcal{C}=2\left|\alpha_{1} \alpha_{4}-\alpha_{2} \alpha_{3}\right|$. Now, we define

$$
x_{ \pm}=\frac{\alpha_{1}^{*} \alpha_{2}+\alpha_{3}^{*} \alpha_{4}}{\mathcal{N}_{ \pm}} \quad y_{ \pm}=\frac{\lambda_{ \pm}-\left(\left|\alpha_{1}\right|^{2}+\left|\alpha_{3}\right|^{2}\right)}{\mathcal{N}_{ \pm}}
$$

where

$$
\lambda_{ \pm}=\frac{1}{2}\left[1 \pm \sqrt{1-\mathcal{C}^{2}}\right] \quad \mathcal{N}_{ \pm}^{2}=\left|\alpha_{1}^{*} \alpha_{2}+\alpha_{3}^{*} \alpha_{4}\right|^{2}+\left|\lambda_{ \pm}-\left(\left|\alpha_{1}\right|^{2}+\left|\alpha_{3}\right|^{2}\right)\right|^{2} .
$$

Now, we consider $2 \times 2$ matrix $u$, whose components $u_{i j}$ are

$$
\begin{aligned}
& u_{11}=\alpha_{1}\left(\frac{\left|x_{+}\right|^{2}}{\sqrt{\lambda_{+}}}+\frac{\left|x_{-}\right|^{2}}{\sqrt{\lambda_{-}}}\right)+\alpha_{2}\left(\frac{x_{+}^{*} y_{+}}{\sqrt{\lambda_{+}}}+\frac{x_{-}^{*} y_{-}}{\sqrt{\lambda_{-}}}\right) \\
& u_{12}=\alpha_{1}\left(\frac{x_{+} y_{+}^{*}}{\sqrt{\lambda_{+}}}+\frac{x_{-} y_{-}^{*}}{\sqrt{\lambda_{-}}}\right)+\alpha_{2}\left(\frac{\left|y_{+}\right|^{2}}{\sqrt{\lambda_{+}}}+\frac{\left|y_{-}\right|^{2}}{\sqrt{\lambda_{-}}}\right) \\
& u_{21}=\alpha_{3}\left(\frac{\left|x_{+}\right|^{2}}{\sqrt{\lambda_{+}}}+\frac{\left|x_{-}\right|^{2}}{\sqrt{\lambda_{-}}}\right)+\alpha_{4}\left(\frac{x_{+}^{*} y_{+}}{\sqrt{\lambda_{+}}}+\frac{x_{-}^{*} y_{-}}{\sqrt{\lambda_{-}}}\right) \\
& u_{22}=\alpha_{3}\left(\frac{x_{+} y_{+}^{*}}{\sqrt{\lambda_{+}}}+\frac{x_{-} y_{-}^{*}}{\sqrt{\lambda_{-}}}\right)+\alpha_{4}\left(\frac{\left|y_{+}\right|^{2}}{\sqrt{\lambda_{+}}}+\frac{\left|y_{-}\right|^{2}}{\sqrt{\lambda_{-}}}\right) .
\end{aligned}
$$

Then Schmidt bases for each party are defined as

$$
\left|i_{A}\right\rangle=\sum_{j=0}^{1} v_{j i}|j\rangle \quad\left|i_{B}\right\rangle=\sum_{k=0}^{1} w_{i k}|k\rangle \quad(i=0,1)
$$

where

$$
v=\left(\begin{array}{ll}
u_{11} & u_{12} \\
u_{21} & u_{22}
\end{array}\right)\left(\begin{array}{ll}
x_{+} & x_{-} \\
y_{+} & y_{-}
\end{array}\right) \quad w=\left(\begin{array}{ll}
x_{+}^{*} & y_{+}^{*} \\
x_{-}^{*} & y_{-}^{*}
\end{array}\right) .
$$

Using Eq. A.4, one can show straightforwardly that $\left|\psi_{2}\right\rangle_{A B}$ reduces to $\left|\psi_{2}\right\rangle_{A B}=$ $\sqrt{\lambda_{+}}\left|0_{A} 0_{B}\right\rangle+\sqrt{\lambda_{-}}\left|1_{A} 1_{B}\right\rangle$. Thus, its CSS $\sigma_{*}$ are simply expressed in terms of the Schmidt bases as

$$
\sigma_{*}=\lambda_{+}\left|0_{A} 0_{B}\right\rangle\left\langle 0_{A} 0_{B}\left|+\lambda_{-}\right| 1_{A} 1_{B}\right\rangle\left\langle 1_{A} 1_{B}\right| .
$$

Applying Eq. 1.1), one can show easily $\mathcal{E}_{R}\left(\left|\psi_{2}\right\rangle\right)=-\lambda_{+} \ln \lambda_{+}-\lambda_{-} \ln \lambda_{-}$, which is exactly the same with EOF. 